\title{
Probabilities of TKR Patients Achieving their Goals
}

\author{
Rajitha Gunaratne ${ }^{1,2^{*}}$, Riaz Khan ${ }^{1-6}$, Daniel Fick ${ }^{1-5}$, Jami llyas ${ }^{3}$, Samantha Haebich ${ }^{3}$ and Anne Smith ${ }^{1}$ \\ ${ }^{1}$ Curtin University, Kent Street, Bentley, WA, 6102, Australia \\ ${ }^{2}$ Australian Institute of Robotic Orthopaedics, 2 Centro Avenue, Subiaco, WA, 6008, Australia \\ ${ }^{3}$ The Joint Studio, Hollywood Medical Centre, 85 Monash Avenue, Nedlands, WA, 6009, Australia \\ ${ }^{4}$ The Virtual Centre for Advanced Orthopaedics, 1155 Union Circle, Denton, Texas 76203, United States of America \\ ${ }^{5}$ University of North Texas, 1155 Union Circle, Denton, Texas 76203, United States of America \\ ${ }^{6}$ University of Notre Dame, 9 Mouat Street, Fremantle, WA, 6959, Australia \\ *Corresponding author: Rajitha Gunaratne, Curtin University, Kent Street, Bentley, WA, 6102, Australia, Tel: 0410990265; Fax: 08 9431 2701; E-mail: \\ gunaratne.rajitha@gmail.com
}

Received date: September 14, 2018; Accepted date: October 03, 2018; Published date: October 08, 2018

Copyright: (c) 2018 Gunaratne R, et al. This is an open-access article distributed under the terms of the Creative Commons Attribution License, which permits unrestricted use, distribution, and reproduction in any medium, provided the original author and source are credited.

\begin{abstract}
Introduction: Prospective total knee replacement patients often enquire about the likelihood that surgery will resolve their knee pain, enable them to walk "normally", and to resume activities important to them. However, considerable variability exists in patient outcomes, which makes it difficult for clinicians to accurately answer these questions, and for patients to make informed decisions. In this study five patient-centred outcomes were explored: pain resolution, walk without limping, perform usual work, ability to kneel, and satisfaction.
\end{abstract}

Significance: The goal of this paper was not to create a fully predictive framework of outcomes after TKR, but to focus on patient-centred goals using only easily measured baseline factors to see if doing this might be achievable and potentially useful in terms of discussion of expectations in shared decision making for surgery. The added value of personalising each outcome, as opposed to providing all patients with a generic probability, was also assessed from the statistical models.

Method: Data from 470 patients was used in multivariable logistic regression analyses to identify independent significant predictors for each goal. Predictors assessed were age, gender, body mass index, preoperative knee function, physical health status and mental health status.

Results: The likelihood of achieving a desirable outcome varied across goals examined. Whilst $82 \%$ of patients were able to walk without a consistent limp, only $32 \%$ could kneel with ease. Furthermore, we identified a consistent pattern where patients with greater preoperative knee function and mental health, had improved odds for attaining each goal. Preoperative physical health and body mass also had some predictive utility.

Conclusion: We found that when assessing the merits of undergoing total knee replacement, consideration of a patient's pre-operative knee function and mental health allows a more accurate prediction of the benefit they may achieve.

Keywords: TKR; Patient education; Patient selection

Abbreviations TKR: Total Knee Replacement; PROMS: Patient Reported Outcome Measures; OKS: Oxford Knee Score; BMI: Body Mass Index; SF12 PCS: Short Form 12 Physical Component Score; SF12 MCS: Short Form 12 Mental Component Score

\section{Introduction}

Prospective total knee replacement (TKR) patients often enquire about the likelihood that surgery will resolve their knee pain, enable them to walk "normally", and to resume activities important to them. As there is considerable variability with regard to these outcomes, it is difficult for clinicians to accurately answer these questions, and for patients to form accurate expectations and make well-informed decisions regarding TKR surgery $[1,2]$.
When evaluating the importance of outcomes, surgeons prioritise joint alignment and stability while patients place more value on vitality and the ability to return to leisure activities [3]. The successful attainment of patient goals is an important determinant of patient satisfaction [3]. Scott et al. [4] identified improvement in walking ability, pain relief, ability to perform activities of daily living, and kneeling to be important outcomes for prospective TKR patients [5]. Goldsmith et al. [6] highlights that unmet informational support about pain, pain management, and recovery trajectory negatively impacts the TKR experience [7].

The literature provides some insight into factors affecting TKR outcomes. High preoperative pain [8] and low scores on preoperative patient reported outcome measures (PROMS) have been associated with worse postoperative outcomes [4,9]. Younger age [9], female gender [10], higher number of comorbidities [8], and worse mental health status [8] have been also associated with poorer outcomes. However, outcomes are reported in broad terms based upon 
multidimensional functional scales. There is little in the literature with regards to the predictive utility for specific functional activities.

This study aimed to identify predictors for five patient-centred goals one year following TKR. A set of easily measured preoperative factors was investigated to quantify the likelihood of each goal being achieved. This information could assist in personalising and improving the accuracy of education delivered at the initial orthopaedic consultation, promote more realistic expectations being set, and ultimately improve patient satisfaction.

\section{Patients and Methods}

\section{Patients}

This study was approved by our institutional ethical review committee. The data of consecutive patients undergoing unilateral primary total knee replacement between September 2009 and September 2015, was extracted from a prospective database of two surgeons with 10-15 years' experience in performing TKR. All patients followed a standardised pathway of care at one institution. Selection was not biased by age, aetiology, implant model or fixation. The preferred surgical approach was medial parapatellar. Exclusion criterion was failure to provide preoperative PROM data.

\section{Selection of important goals}

The first author reviewed the literature to identify goals important to TKR patients, and then discussed these with an expert clinical panel consisting of two surgeons and two physiotherapists, each with over 15 years' experience. Oxford Knee Score (OKS) and satisfaction were routinely captured on the prospective database, and important goals were identified from the available items in the OKS [5]. The five goals selected for investigation were: (i) experiences very mild or no knee pain, (ii) has no limp or limps just at first, (iii) has little or no pain interference with usual work, (iv) can kneel with little or no difficulty, and (v) reports being satisfied or very satisfied with their TKR outcome overall. Satisfaction level was established via a five point Likert scale. The desirable responses (e.g. very satisfied and satisfied) were combined and given a binary value for statistical analyses.

\section{Selection of predictive variables}

Exploring the available literature identified preoperative variables known to influence postoperative TKR outcomes. The panel considered only variables that could easily be obtained preoperatively during a consultation and selected six variables to investigate. These were age, gender, body mass index (BMI), preoperative knee function, physical health status and mental health status. Age on the day of the operation was measured in years. Gender was identified as male or female. BMI was calculated from weight and height. Knee function was assessed using the 12-item OKS, and scored whereby 48 represents the best possible outcome [5]. Health status was assessed via the validated multipurpose self-reported Short Form 12, which gives a physical component score (SF12 PCS) and mental component score (SF12 MCS) [11].

\section{Statistical analysis}

Univariate logistic regression analysis was undertaken to obtain unadjusted odds ratios and associated $95 \%$ confidence intervals and $\mathrm{p}$ values for each potential baseline predictor. Then, multivariate logistic regression analyses were conducted for each of the five binary goals. Initially, this included all variables significant at $\mathrm{p}<0.10$ on univariate analysis. Then, those variables not significant in the multivariate model at $\mathrm{p}<0.05$ were dropped after confirming the absence of confounding effects of the deleted variable by ensuring retained parameter estimates did not change by more than $20 \%$ [12]. The final models retained only variables significant at $\mathrm{p}<0.05$.

Model fit was assessed by Nagelkerke's $\mathrm{R}^{2}$, which although not analogous to proportion of variance explained in linear regression, is indicative of the model's improvement in prediction over a null model with no predictors. Values range from 0 to 1 , with higher values indicating better fit. Model discrimination was assessed by the area under the receiver operator curve (ROC), for which a value of 0.5 indicates that the model discriminates no better than chance and a value of one indicates that the model discriminates perfectly [13]. Residual diagnostics were examined to detect outlying covariate patterns with undue influence on the model. To assist clinical interpretation, predicted probabilities from the model are presented for various covariate patterns.

\section{Results}

\section{Sample}

The sample consisted of 470 unilateral TKR patients ( $46 \%$ male) with a mean age of 68 years (Table 1). One year following TKR, the percentage of patients who achieved a desirable outcome for each goal varied. The goal to have no limp or just at first was achieved by $82 \%$ of patients; however only $32 \%$ reported they could kneel without moderate difficulty. The probability for the cohort to achieve each goal is detailed in Table 2 .

\begin{tabular}{|l|l|}
\hline & Mean (SD) \\
\hline Age (years) & $67.7(9.6)$ \\
\hline Body Mass Index (kg/m2) & $31.2(5.8)$ \\
\hline Knee function (OKS) & $21.5(7.8)$ \\
\hline Physical health status (SF12 PCS) & $32.0(7.7)$ \\
\hline Mental Health status (SF12 MCS) & $49.3(12.0)$ \\
\hline
\end{tabular}

Table 1: Preoperative characteristics of participants $(n=470)$.

\begin{tabular}{|l|l|}
\hline Goal & $\%$ \\
\hline Very mild or no pain & 67 \\
\hline No limp or just at first & 82 \\
\hline No or little pain interference with usual work & 77 \\
\hline Little of no difficulty kneeling & 32 \\
\hline Satisfied or very satisfied & 79 \\
\hline
\end{tabular}

Table 2: Percentage of patients achieving goals at 12 months.

\section{Predictors of goals}

Predictors of an increased (or decreased) likelihood of goal achievement also varied by across the five goals. The univariate and 
Citation: Gunaratne R, Khan R, Fick D, llyas J, Haebich S, et al. (2018) Probabilities of TKR Patients Achieving their Goals. J Arthritis 7: 275. doi:10.4172/2167-7921.1000275

Page 3 of 7

multivariate predictors are listed in Table 3. The multivariate analyses can be interpreted using odd ratios. For example, for each one-point increase in SF12 MCS and OKS, the odds of reporting 'mild or no pain' at one year increased by $2 \%$ and $3 \%$ respectively. For each one-point increase in OKS, it was estimated that the odds of reporting 'no limp or just at first' increased by $10 \%$. For each one-point increase in OKS, SF12 PCS and SF12 MCS, it was estimated that the odds of reporting 'little or no interference with usual work' increased by 7\%, $4 \%$ and $5 \%$ respectively. For each one-point increase in BMI, it was estimated that the odds of reporting "kneeling with little or no difficulty" decreased by $5 \%$ and for each one-point increase in SF12 MCS and OKS, it was estimated that the odds of achieving this outcome increased by $3 \%$ and $6 \%$ respectively. Lastly, for each one-point increase in SF12 MCS, it was estimated that the odds of a patient being "satisfied" increased by $3 \%$.

Common predictors of TKR goal achievement were preoperative knee function (OKS) and mental health status (SF12 MCS). Better Oxford knee scores positively influenced all outcomes except satisfaction $(\mathrm{p}<0.001)$. Better Short-form 12 mental health status positively impacted all outcomes except limping $(\mathrm{p}<0.001)$.

\begin{tabular}{|c|c|c|c|c|c|c|c|c|}
\hline & $\begin{array}{l}\text { Univariate OR } \\
(95 \% \mathrm{Cl}), \mathrm{p}\end{array}$ & & $\begin{array}{l}\text { Mutivariate OR } \\
(95 \% \mathrm{Cl}), \mathrm{p}\end{array}$ & & 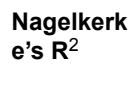 & ROC AUC & $\begin{array}{l}\operatorname{Pr} \text { (at 1SD below } \\
\text { mean for all } \\
\text { predictorsa) }\end{array}$ & $\begin{array}{l}\mathrm{Pr} \text { (at 1SD } \\
\text { above mean for } \\
\text { all predictorsa) }\end{array}$ \\
\hline & OR $(95 \% \mathrm{Cl})$ & p & OR $(95 \% \mathrm{Cl})$ & $\mathbf{p}$ & & & & \\
\hline \multicolumn{9}{|c|}{ Goal 1: Experiences very mild or no knee pain } \\
\hline Male sex & $1.08(0.74-1.59)$ & 0.691 & & & & & & \\
\hline Age (yrs) & $1.02(1.00-1.04)$ & 0.11 & & & & & & \\
\hline BMI & $1.00(0.96-1.03)$ & 0.893 & & & & & & \\
\hline SF12 PCS & $1.03(1.00-1.05)$ & 0.051 & & & & & & \\
\hline SF12 MCS & $1.03(1.01-1.05)$ & $<0.001$ & $1.02(1.00-1.04)$ & 0.012 & & & & \\
\hline OKS & $1.05(1.02-1.08)$ & $<0.001$ & $1.03(1.01-1.06)$ & 0.017 & & & & \\
\hline $\begin{array}{l}\text { Model fit/ } \\
\text { discrimination }\end{array}$ & & & & & 0.059 & 0.623 & 0.54 & 0.78 \\
\hline \multicolumn{9}{|c|}{ Goal 2: Has no limp or limps just at first } \\
\hline Male sex & $0.90(0.57-1.44)$ & 0.663 & & & & & & \\
\hline Age (yrs) & $1.02(1.00-1.05)$ & 0.092 & & & & & & \\
\hline BMI & $1.01(0.96-1.05)$ & 0.812 & & & & & & \\
\hline SF12 PCS & $1.05(1.01-1.09)$ & 0.007 & & & & & & \\
\hline SF12 MCS & $1.04(1.02-1.06)$ & $<0.001$ & & & & & & \\
\hline OKS & $1.10(1.06-1.14)$ & $<0.001$ & $1.10(1.06-1.14)$ & $<0.001$ & & & & \\
\hline $\begin{array}{l}\text { Model fit/ } \\
\text { discrimination }\end{array}$ & & & & & 0.101 & 0.686 & 0.72 & 0.91 \\
\hline \multicolumn{9}{|c|}{ Goal 3: Has little or no pain interference with usual work } \\
\hline Male sex & $1.39(0.90-2.14)$ & 0.139 & & & & & & \\
\hline Age (years) & $1.01(0.99-1.04)$ & 0.223 & & & & & & \\
\hline BMI & $1.00(0.97-1.04)$ & 0.827 & & & & & & \\
\hline SF12 PCS & $1.08(1.05-1.12)$ & $<0.001$ & $1.07(1.02-1.12)$ & 0.003 & & & & \\
\hline SF12 MCS & $1.05(1.07-1.15)$ & $<0.001$ & $1.04(1.02-1.06)$ & 0.001 & & & & \\
\hline OKS & $1.11(1.07-1.15)$ & $<0.001$ & $1.05(1.01-1.10)$ & 0.015 & & & & \\
\hline $\begin{array}{l}\text { Model fit/ } \\
\text { discrimination }\end{array}$ & & & & & 0.189 & 0.74 & 0.5 & 0.95 \\
\hline \multicolumn{9}{|c|}{ Goal 4: Kneels with little or no difficulty } \\
\hline Male sex & $1.56(1.05-2.30)$ & 0.026 & & & & & & \\
\hline
\end{tabular}




\begin{tabular}{|c|c|c|c|c|c|c|c|c|}
\hline Age (years) & $1.00(0.98-1.02)$ & 0.865 & & & & & & \\
\hline BMI & $0.93(0.90-0.97)$ & $<0.001$ & $0.95(0.91-0.99)$ & 0.011 & & & & \\
\hline SF12 PCS & $1.06(1.03-1.09)$ & $<0.001$ & & & & & & \\
\hline SF12 MCS & $1.04(1.03-1.06)$ & $<0.001$ & $1.03(1.01-1.05)$ & 0.002 & & & & \\
\hline OKS & $1.07(1.05-1.10)$ & $<0.001$ & $1.06(1.03-1.09)$ & $<0.001$ & & & & \\
\hline $\begin{array}{l}\text { Model fit/ } \\
\text { discrimination }\end{array}$ & & & & & 0.144 & 0.691 & 0.12 & 0.56 \\
\hline \multicolumn{9}{|c|}{ Goal 5: Is satisfied or very satisfied with TKR surgery outcome } \\
\hline Male sex & $0.83(0.53-1.31)$ & 0.418 & & & & & & \\
\hline Age (years) & $1.00(0.98-1.03)$ & 0.848 & & & & & & \\
\hline BMI & $1.00(0.96-1.04)$ & 0.932 & & & & & & \\
\hline SF12 PCS & $1.01(0.98-1.04)$ & 0.638 & & & & & & \\
\hline SF12 MCS & $1.03(1.01-1.05)$ & 0.001 & $1.03(1.01-1.05)$ & 0.001 & & & & \\
\hline OKS & $1.04(1.01-1.08)$ & 0.006 & & & & & & \\
\hline $\begin{array}{l}\text { Model fit/ } \\
\text { discrimination }\end{array}$ & & & & & 0.04 & 0.61 & 0.72 & 0.85 \\
\hline
\end{tabular}

Table 3: Results of univariate and multivariate logistic regression models for each outcome at 12 months.

\section{Personalising predictions}

Model fit and discrimination, calculated by the Nagelkerke's $\mathrm{R}^{2}$ value and AUC, is presented in Table 3. The added value of "personalising" the probabilities using the predictive variables was greatest for the goal to have no or little pain interference with usual work ( $\mathrm{R}^{2}$ 0.189, AUC 0.740). Being one standard deviation above the mean as opposed to one standard deviation below on all three predictor variables (OKS, SF12 MCS and PCS) improved the goal achievement likelihood by $45 \%$ from $50 \%$ to $95 \%$ suggesting providing personalised predictions for this goal would be clinically useful. Without "personalising" by accounting for baseline status, the predicted probability for any patient for this goal reduces to the population proportion achieving this outcome of $77 \%$ (Table 2).

In contrast, the logistic regression model for the goal to be 'Satisfied' had the lowest $\mathrm{R}^{2}$ value (0.04) and the lowest AUC (0.610), meaning personalised prediction for this goal is the least clinically useful. The probability of being satisfied only varied from 0.72 to 0.85 for those patients one standard deviation below/above the mean respectively on the one significant predictor SF12 MCS.

\section{Discussion}

Current methods for setting goals and expectation require clinicians to be well versed in the orthopaedic literature pertaining to past and emerging outcome reporting, and to compliment this with the knowledge they derive from observing progress of their own patients. Attention should be given to outcomes which patients deem important and on which they base their satisfaction. Presenting the likelihood of being able to easily perform key activities is a meaningful way to educate patients in terms that they can easily comprehend

This discussion firstly examines the probability of attaining a desirable outcome, and then explores the relative merits of further "personalising" these probabilities using identified predictors.

\section{Generalised goal achievement probabilities}

Our examination of a unilateral TKR cohort found $67 \%$ experienced good pain relief at one year, whereas $33 \%$ reported pain levels as mild, moderate or severe. This matches findings of Parvizi et al. [13] who reported that $33 \%$ of TKRs had lingering pain. Similarly, O'Brien et al. [9] found $25.8 \%$ of TKR patients reported moderate or severe pain at three months postoperatively.

A clear majority (82\%) of our cohort was able to walk without a limp. Gait studies have identified that after TKR patients walk more slowly with asymmetrical support moments [14]. Whilst improvements occur following surgery, it is difficult to determine whether gait can be defined as normal, and a significant gap is noted in the literature regarding the prevalence of limping following TKR. However, the question "When will I walk normally?" was identified as being amongst the top four concerns for surgical candidates before TKR (14).

The goal to experience minimal interference with usual or household work was achieved by $77 \%$ of patients. In comparison, more than $30 \%$ of patients in the Parvizi et al. [13] cohort had some impairment with daily activities. A likely explanation lies in the line of questioning in this study, which encompassed difficulty in getting in 
and out of a car (38\%), getting up and down from a chair (31\%), and climbing up and down stairs (54\%).

Kneeling is an important function required for many activities. Inability to kneel after knee surgery is a frequent cause of dissatisfaction [15]. Almost a third (32\%) of the cohort examined reported being able to kneel with relative ease. While this percentage appears low, it encompasses only those able to perform the task easily or with just a little difficulty, as distinct from those who can kneel but find it moderately or extremely difficult. Most studies report ability verses inability to kneel [16]. Schai et al. [17] found that fear of harming the prosthesis and lack of information prevented kneeling in $49 \%$ of patients. Hassabella et al. [18] studied 38 TKRs and $47 \%$ had a perceived inability to kneel, whereas when attempted, only $24 \%$ were unable to perform the task. Interestingly, a study by White et al. [19] showed that $72 \%$ of patients were able to kneel after a targeted education program.

The majority $(79 \%)$ of our cohort was either satisfied or very satisfied. This prevalence is consistent with rates reported in the literature $[7,20,21]$. High BMI has been linked with poor satisfaction [22], however ours and other studies show no association [23].

\section{Personalising probabilities based on Predictors}

When looking to "personalise" the probability of achieving a desired goal, preoperative OKS and mental health status were the two most predictive variables, and these findings are consistent with the literature. Preoperative knee pain and function strongly influenced the probability that a desirable surgical outcome would be achieved in all of the patient-centred goals we investigated, excepting satisfaction in the multivariate analysis. A plausible explanation for the association with preoperative OKS may involve pain centralisation and disuse atrophy that are associated with chronic disease and lower physical functioning. This pain and disability persist despite an anatomically well-aligned TKR [24].

Preoperative mental health status (SF12 MCS) was significantly associated with all outcomes and was also the only variable predictive of satisfaction on multivariate analysis. This finding is supported by Brander et al. [25] who found preoperative anxiety and depression to be one of the strongest predictors of pain relief and patient satisfaction. Similarly, other researchers have found that arthroplasty patients with depression have higher postoperative pain scores and analgesia use [26], as well as worse subjective and objective outcomes measures [8]. These findings highlight a need to acknowledge the impact of mental health when educating and setting expectations for people considering TKR. Psychiatric illness is more prevalent in patients undergoing arthroplasty than the general population [27]; depression is common in patients with chronic musculoskeletal pain [28] and $23-26 \%$ of patients presenting for TKR have depression [29]. The World Health Organisation has predicted that by 2030, depression will continue to rise and account for the highest level of disability conferred to any physical or mental disorder in the world [30]. It is important to note, however that SF12 is a measure of mental wellbeing and is not diagnostic for a mental health disorder. Mental health can encompass not only affective factors but also cognitive factors like fear, self-efficacy and illness perceptions. These perceptions have also been found to be important prognostic factors for pain and disability in knee osteoarthritis $[31,32]$.

Physical health status before surgery predicted achieving 'little or no pain interference with usual household tasks'. Physically able individuals are more likely to get better outcomes and conversely, those less physically able, may be deconditioned, limited by other jointrelated problems, or simply follow habitual patterns, and so may not achieve the same post-operative benefit.

Lastly, we also found BMI to predict the goal of kneeling. A link with BMI and kneeling ability after TKR has not been reported in the orthopaedic literature to our knowledge, however a study of an older adult population by Dowd and Zajacova found that obese individuals were twice as likely to report severe limitations in stooping, crouching or kneeling than those of a normal BMI range [33]. In contrast to our multivariate analyses, Hassabella et al. [19] reported kneeling ability after TKR in men to be significantly better than in women $(\mathrm{p}<0.001)$.

Gender and age were predictive on univariate analyses, but did not add value to the multivariate models and would appear less helpful when tailoring expectations for TKRs. This reflects inconsistencies found in the literature whereby some studies show older patients and females to have better outcomes [25], while other studies have shown that younger patients $[4,33]$ and males fare better $[10,19]$.

A limitation of our study is that the outcomes selected by our panel likely do not comprehensively cover all goals that might be important to patients. Also, the definition of each goal may not precisely reflect patients' aims. Similarly, the study may not have captured all predictive variables. Co-morbidities [8,34], sociodemographic factors [30] and level of preoperative pain have been noted to influence overall TKR functional scores. Co-morbidities and sociodemographic factors were not included because of the difficultly to grade these quickly within an initial consult. Pain level was not included specifically since this was encompassed within the OKS. We also relied on subjective reporting which may not accurately reflect the true attainment of the goals studied. There is a tendency for patients with more optimistic expectations to report postoperatively that these were poorly met [35]. Several researchers have showed differences between the perceived and actual ability to kneel after total knee arthroplasty $[17,19,36]$. Conversely, post-operative responses may be overstated, in an attempt to please the surgeon. Mancuso et al. [37] found increased age and poorer pre-operative functional ability to be associated with higher pre-operative expectations. Lastly, our use of historical data from two surgeons may not extrapolate to future outcomes or different geographic communities.

A strength of our study is in the examination of patient-centred factors using prospectively collected data. We explored both the average probability of reaching a goal, as well as variables that can attune the expectations for individuals or a categorised group (such as low verses high preoperative function). Further "personalising" patient expectations was found worthwhile for some outcomes but not for others. In particular, personalising the likelihood of satisfaction was not very useful, in terms of the predictors we investigated.

In contrast, existing nomograms are usually based on 'responder' criteria based on change on self-reported pain and function questionnaires. Whilst these can be helpful for surgeons [38], concepts such as risk and probability are often difficult for patients and the general public to understand and relate to personally. The way in which personalised probabilities are quantified, communicated and evaluated should be the subject of further investigation.

The goal of this paper was not to create a fully predictive framework of outcomes after TKR, but to focus on the prediction of patientcentred goals using only easily measured baseline factors to see if doing 
this might be achievable and potentially useful in terms of discussion of expectations in shared decision making for surgery.

\section{Conclusion}

This novel study identifies the probability that TKR patients will achieve a desirable outcome for five specific goals. Six easily assessable preoperative measures are also investigated to determine their utility as personalised predictors for use in shared decision-making. Patients' preoperative knee function and mental health scores were shown to best predict patient-centric goals at one-year following TKR surgery. Our results are likely to improve quality of patient education, help set more accurate patient expectations, and in turn increase overall satisfaction for prospective TKR patients.

\section{Compliance with Ethical Standards}

Conflict of Interest: The authors declare that they have no conflict of interest.

Funding: There is no funding source.

Ethics approval: Yes

\section{References}

1. Hamilton D, Henderson GR, Gaston P, MacDonald D, Howie C, et al. (2012) Comparative outcomes of total hip and knee arthroplasty: a prospective cohort study. Postgrad Med J 88: 627-631.

2. Noble PC, Conditt MA, Cook KF, Mathis KB (2006) The John Insall Award: patient expectations affect satisfaction with total knee arthroplasty. Clin Orthop Relat Res 452: 35-43.

3. Janse AJ, Gemke RJ, Uiterwaal CS, van der Tweel I, Kimpen JL, et al. (2004) Quality of life: patients and doctors don't always agree: a metaanalysis. J Clin Epidemiol 57: 653-661.

4. Scott CEH, Bugler KE, Clement ND, MacDonald D, Howie CR, et al. (2012) Patient expectations of arthroplasty of the hip and knee. J Bone Joint Surg Br 94: 974-981.

5. Dawson J, Fitzpatrick R, Murray D, Carr A (1998) Questionnaire on the perceptions of patients about total knee replacement. J Bone Joint Surg Br 80: 63-69.

6. Goldsmith LJ, Suryaprakash N, Randall E, Shum J, MacDonald V, et al. (2017) The importance of informational, clinical and personal support in patient experience with total knee replacement: a qualitative investigation. BMC Musculoskelet Disord 18: 127.

7. Scott CEH, Howie CR, MacDonald D, Biant LC (2010) Predicting dissatisfaction following total knee replacement. J Bone Joint Surg $\mathrm{Br} 92$ : 1253-1258.

8. Lingard EA KJ, Wright EA, Sledge CB (2004) Predicting the outcome of total knee arthroplasty. J Bone Joint Surg Am 86-A: 2179-2186.

9. O'Brien S BD, Doran E, Beverland DE (2009) Comparison of hip and knee arthroplasty outcomes at early and intermediate follow-up. Orthopedics 32: 170-176.

10. Bonnin MP, Basiglini L, Archbold HA (2011) What are the factors of residual pain after uncomplicated TKA? Knee Surg Sports Traumatol Arthrosc 19: 1411-1417.

11. Brazier J, Roberts J (2004) The estimation of a preference-based measure of health from the SF-12. Med Care 42: 851-859.

12. Hosmer DW, Lemeshow S (2000) Applied logistic regression. Wiley, New York.

13. Parvizi J, Nunley RM, Berend KR, Lombardi AV Jr, Ruh EL, et al. (2014) High level of residual symptoms in young patients after total knee arthroplasty. Clin Orthop Relat Res 472: 133-137.

14. Yoshida Y, Mizner R, Ramsey DK, Snyder-Mackler L (2008) Examining outcomes from total knee arthroplasty and the relationship between quadriceps strength and knee function over time. Clin Biomech 23: 320-328.

15. Macario A, Schilling P, Rubio R, Bhalla A, Goodman S (2003) What questions do patients undergoing lower extremity joint replacement surgery have? BMC Health Serv Res 3: 11.

16. Wilkens KJ, Duong LV, McGarry MH, Kim WC, Lee TQ (2007) Biomechanical effects of kneeling after total knee arthroplasty. J Bone Joint Surg Am 89: 2745-2751.

17. Schai PA, Gibbon AJ, Scott RD (1999) Kneeling ability after total knee arthroplasty. Perception and reality. Clin Orthop Relat Res 367: 195-200.

18. Hassaballa M, Porteous A, Newman JH (2004) Observed kneeling ability after total, unicompartmental and patellofemoral knee arthroplasty: perception versus reality. Knee Surgery Sports Traumatology Arthroscopy 12: 136-139.

19. White L, Stockwell T, Hartnell N, Hennessy M, Mullan J (2016) Factors preventing kneeling in a group of pre-educated patients post total knee arthroplasty. J Orthopaed Traumatol 17: 333-318.

20. Baker PN VdMJ, Lewsey J, Gregg PJ (2007) The role of pain and function in determining patient satisfaction after total knee replacement. J Bone Joint Surg Br 7: 893-900.

21. Nam D, Nunley RM, Barrack RL (2014) Patient dissatisfaction following total knee replacement: a growing concern? Bone Joint J 96-B: 96-100.

22. Fisher DA, Dierckman B, Watts MR, Davis K (2007) Looks good but feels bad: factors that contribute to poor results after total knee arthroplasty. J Arthroplasty 22: $39-42$.

23. Judge A, Arden NK, Cooper C, Kassim Javaid M, Carr AJ, et al. (2012) Predictors of outcomes of total knee replacement surgery. Rheumatology (Oxford) 51: 1804-1813.

24. Arendt-Nielsen L, Nie H, Laursen MB, Laursen BS, Madeleine P, et al. (2010) Sensitization in patients with painful knee osteoarthritis. Pain 149: 573-581.

25. Brander V, Gondek S, Martin E, Stulberg SD (2007) Pain and depression influence outcome 5 years after knee replacement surgery. Clin Orthop Relat Res 464: 21-26.

26. Rakel BA, Blodgett NP, Bridget Zimmerman M, Logsden-Sackett N, Clark C, et al. (2012) Predictors of postoperative movement and resting pain following total knee replacement. Pain 153: 2192-2203.

27. Browne JA, Sandberg BF, D'Apuzzo MR, Novicoff WM (2014) Depression Is Associated With Early Postoperative Outcomes Following Total Joint Arthroplasty: A Nationwide Database Study. J Arthroplasty 29: 481-483.

28. Magni G, Moreschi C, Rigatti-Luchini S, Merskey H (1994) Prospective study on the relationship between depressive symptoms and chronic musculoskeletal pain. Pain 56: 289-297.

29. Riddle DL, Wade JB, Jiranek WA (2010) Major Depression, Generalized Anxiety Disorder, and Panic Disorder in Patients Scheduled for Knee Arthroplasty. J Arthroplasty 25: 581-588.

30. Manicavasagar V (2012) A Review of depression diagnosis and management.

31. Erfani T, Keefe F, Bennell K, Chen J, Makovey J, et al. (2015) Psychological Factors and Pain Exacerbation in Knee Osteoarthritis: A Web Based Case-Crossover Study. Rheumatology (Sunnyvale) S6: 005.

32. Kittelson AJ, George SZ, Maluf KS, Stevens-Lapsley JE (2014) Future Directions in Painful Knee Osteoarthritis: Harnessing Complexity in a Heterogeneous Population. Phys Ther 94: 422-432.

33. Dowd JB, Zajacova A (2015) Long-term obesity and physical functioning in older Americans. Int J Obes 39: 502-507.

34. Escobar A, Quintana JM, Bilbao A, Azkarate J, Guenaga JI, et al. (2007) Effect of patient characteristics on reported outcomes after total knee replacement. Rheumatology (Oxford) 46: 112-119.

35. Mannion AF, Kämpfen S, Munzinger U, Kramers-de QI (2009) The role of patient expectations in predicting outcome after total knee arthroplasty. Arthritis Res Ther 11: R139.

36. Palmer SH SC, Maguire J, Parish EN, Cross MJ (2002) Ability to knee after total knee replacement. J Bone Joint Surg Br 84: 220-222. 
Citation: Gunaratne R, Khan R, Fick D, llyas J, Haebich S, et al. (2018) Probabilities of TKR Patients Achieving their Goals. J Arthritis 7: 275. doi:10.4172/2167-7921.1000275

Page 7 of 7

37. Mancuso CA, Sculco TP, Salvati EA (2003) Patients with poor preoperative functional status have high expectations of total hip arthroplasty. J Arthroplasty 18: 872-878.

38. Dowsey MM, Spelman T, Choong PF (2016) Development of a Prognostic Nomogram for Predicting the Probability of Nonresponse to
Total Knee Arthroplasty 1 Year After Surgery. J Arthroplasty 31: 1654-1660. 